\title{
Fios de Polidioxanona e Toxina Botulínica como alternativa no tratamento da Paralisia Facial de Bell: relato de experiência
}

\author{
Polydioxanone and Botulinum Toxin threads as an alternative in the treatment of Facial Bell's \\ Palsy: experience report \\ Hilos de Polidioxanona y Toxina Botulínica como alternativa en el tratamiento de la Parálisis Facial \\ de Bell: informe de experiencia
}

\section{Resumo}

A Paralisia de Bell afeta o sétimo nervo craniano que gera perda da função dos músculos faciais unilateralmente. O presente estudo tem como objeto relatar a experiência vivenciada em um tratamento alternativo da paralisia de Bell. Trata-se de um relato de experiência que descreve o acompanhamento de uma paciente com síndrome de Bell, vivenciado em uma clínica particular, Palmas - Tocantins, Brasil. Durante o período entre outubro de 2020 e setembro de 2021 foram realizados 13 sessões com a paciente utilizando a toxina botulínica e fios de Polidioxanona, onde podese perceber uma melhoria na motilidade muscular e redução na assimetria. O diagnóstico é clínico e também de exclusão onde deve-se realizar um exame físico detalhado, o tratamento envolve o uso de medicamentos, métodos físicos e até cirurgia. $\mathrm{O}$ tratamento alternativo empregado no caso relatado se mostrou bastante eficaz, melhorando a estética facial da paciente e sua autoestima.

Palavras-chave: Paralisia; Estética; Polidioxanona.

\begin{abstract}
Bell's Palsy affects the seventh cranial nerve which unilaterally causes loss of function of the facial muscles. This study aims to report the experience of an alternative treatment for Bell's palsy. This is an experience report that describes the follow-up of a patient with Bell syndrome, experienced in a private clinic in the state of Tocantins. During the period between October 2020 and September 2021, 13 sessions were performed with the patient using botulinum toxin and polydioxanone threads, where an improvement in muscle motility and a reduction in asymmetry can be seen. The diagnosis is clinical and also exclusionary, where a detailed physical examination must be performed, the treatment involves the use of medication, physical methods and even surgery. The alternative treatment used in the reported case proved to be quite effective, improving the patient's facial esthetics and self-esteem.
\end{abstract}

Keywords: Paralysis; Esthetics; Polydioxanona.

\section{Resumen}

La parálisis de Bell afecta el séptimo par craneal, lo que provoca unilateralmente la pérdida de función de los músculos faciales. Este estudio tiene como objetivo informar la experiencia de un tratamiento alternativo para la parálisis de Bell. Este es un informe de experiencia que describe el seguimiento de un paciente con síndrome de Bell, experimentado en 
una clínica privada en el estado de Tocantins. Durante el período comprendido entre octubre de 2020 y septiembre de 2021 se realizaron 13 sesiones con el paciente utilizando toxina botulínica e hilos de polidioxanona, donde se aprecia una mejora de la motilidad muscular y una reducción de la asimetría. El diagnóstico es clínico y también excluyente, donde se debe realizar un examen físico detallado, el tratamiento implica el uso de medicación, métodos físicos e incluso cirugía. El tratamiento alternativo utilizado en el caso reportado resultó ser bastante efectivo, mejorando la estética facial y la autoestima del paciente.

Palabras clave: Parálisis; Estética; Polidioxanona.

\section{Introdução}

A Paralisia de Bell (PB) provoca problemas estéticos e funcionais o que afeta diversos aspectos da vida da pessoa trata se de uma doença do nervo facial que gera perda da função de músculos da face na maioria dos casos ocorre de forma súbita e unilateral (Santos, et al., 2020).

Também conhecida por paralisia idiopática facial afeta o sétimo nervo craniano e mesmo que sua etiologia não seja confirmada existe alguns fatores de risco como infecção pelo vírus herpes simplex e pertençam e diabetes gravidez e o puerpério sendo estimado maior acometimento pela doença em mulheres (Belém, et al., 2021).

Existem casos de pacientes que se recuperam totalmente enquanto outros desenvolvem hipertonia, hipercinesia ou sincinesia (Thien, et al., 2019).

Segundo Lima, et al., (2020), a Toxina Botulínica (TB) torna-se uma alternativa para otratamento da paralisia de bell, devendo ser ininioado de maneira precoce.

Estudo realizado em 2020 demonstrou um resultado satisfatório mediante o uso da toxina botulínica para a organização facial do paciente tendo benefícios além da estética melhorando ainda auto estima e consequentemente o convívio social do paciente (Santos, et al., 2020).

A TB consiste em uma substância amplamente utilizada em procedimentos estéticos, essa interage com a placa das terminações neuromusculares e pode ser utilizada em diferentes idades de ambos os sexos, sendo capaz de promover harmonia e melhoria da estética facial (Brito \& Barbosa, 2020).

A TB trata-se de um metabólito produzido pela bactéria Clostridium bolutinum que promove o relaxamento muscular, seu uso possui eficácia comprovada no auxílio de correções faciais e alívio de rugas sendo um procedimento estético não cirúrgico e com raras respostas imunológicas. Dentre os efeitos adversos que podem surgir que geralmente são leves destaca-se o desconforto podendo haver ainda edema, dor, eritema, equimose e cefaleias (Brito \& Barbosa, 2020).

O primeiro relato do uso da toxina botulínica em tratamento foi datado em 1970 onde a substância foi utilizada na medicina para corrigir o estrabismo e até a atualidade observa seu uso em diferentes condições associadas a distonias musculares entre outras (Fujita \& Hurtado, 2019).

A resposta clínica ao tratamento com TB sofrem alterações de pessoa para pessoa levando em consideração a idade e sexo, os efeitos clínicos podem ser observado dentro de 24 a 72 horas no entanto já após 6 horas da aplicação da substância o músculo começa a sofrer paralisia a dose utilizada varia em função da região a ser tratada (Fujita \& Hurtado, 2019).

Dentro da área estética existe uma gama de procedimentos não cirúrgicos que auxiliam no rejuvenescimento facial e em meio a esse cenário surgem os fios de sustentação facial que reposicionam os tecidos faciais, dente eles encontra-se os fios de PDO (Bortolozo, et al., 2021).

Assim a pesquisa em questão justifica-se dada a importância de se buscar evidências que auxiliam no tratamento da PB, afim de encontrar novas alternativas para a recuperação do paciente, proporcionando melhora nos sintomas e consequentemente na qualidade de vida.

O presente estudo tem como objeto relatar a experiência vivenciada em um tratamento alternativo da paralisia de Bell. 


\section{Metodologia}

Trata-se de um relato de experiência que descreve o acompanhamento de uma paciente com síndrome de Bell, vivenciado em uma clínica particular, Palmas - Tocantins, Brasil. Os dados foram coletados mediante observação da paciente.

O presente estudo apresenta-se de forma qualitativa, onde os resultados obtidos, descrevem a evolução da paciente e as condutas tomadas diante do caso. As informações foram anotadas em uma ficha própria do estabelecimento afim de proporcionar um melhor acompanhamento do trabalho desenvolvido durante os atendimento a essa paciente que ocorreram no período entre outubro de 2020 e setembro de 2021.

Por se tratar de relato de experiência não há a necessidade de um parecer ético, ainda assim mediante a Resolução ${ }^{\circ}$ 466/12 que dispõe sobre pesquisas envolvendo seres humanos e os aspectos éticos e legais, no qual diz que todos os pesquisadores devem dar garantias de que os dados serão utilizados apenas para fins cien1tíficos, preservando a privacidade e confidencialidade (Brasil, 2012), o nome da paciente foi criado de forma fictícia para preservar a identidade da mesma e garantir a privacidade.

\section{Resultados}

\section{Histórico da paciente:}

Ana, sexo feminino, 37 anos, 2 filhos, diagnóstico de paralisia de bell, nega uso anterior de toxina botulínica, preenchimento facial e cirurgias estéticas na face. Nega alergias, doenças preexistentes e cardíacas.

\section{Tratamentos:}

Durante o período entre outubro de 2020 e setembro de 2021 foram realizados 13 sessões com a paciente, descritos de acordo com a Tabela 1 apresentada a seguir:

Tabela 1: Procedimentos estéticos realizados.

\begin{tabular}{|c|c|}
\hline Data & Procedimento \\
\hline 24/10/2020 & Aplicação de toxina- 56 pontos \\
\hline 13/11/2020 & Retoque de Botox- 20 und. \\
\hline $02 / 12 / 2020$ & Skinbooster (ácido hialurônico + vitamina c + Dimetilaminoetanol (DMAE)) \\
\hline 19/12/2020 & $\begin{array}{l}\text { Preenchimento malar } 2,5 \mathrm{ml} \text { de juvederma + MD codes de bigode chinês + Skinbooster (Ácido } \\
\text { hialurônico + DMAE + ácido tranexâmico) }\end{array}$ \\
\hline $30 / 01 / 2021$ & Preenchimento labial \\
\hline 05/02/2021 & 5 fios especulados do lado esquerdo \\
\hline $10 / 04 / 2021$ & 5 fios especulados ( 3 lado direito e 2 lado esquerdo) \\
\hline 24/04/2021 & $\begin{array}{l}\text { Preenchimento labial (1 } 1 \mathrm{ml}) \text { juvederma; Rinomodelação da ponta nasal (3 und.); Aplicação de Botox } \\
\text { (50 und.) }\end{array}$ \\
\hline $12 / 05 / 2021$ & Retoque de Botox- 30 und. \\
\hline 18/05/.2021 & Retoque de lábios \\
\hline 29/05/2021 & $\begin{array}{l}2 \text { fios especulados lado direito }+4 \text { fios especulados lado esquerdo }+6 \text { fios lisos bigode chinês, } \\
\text { xantelasma bilateral }\end{array}$ \\
\hline 19/06/2021 & $\begin{array}{l}3 \text { fios especulados lado direito }+3 \text { fios especulados lado esquerdo }+8 \text { fios lisos (4 lado direito e } 4 \text { lado } \\
\text { esquerdo) bigode chinês. }\end{array}$ \\
\hline 25/09/2021 & 6 fios PDO + Brow lift \\
\hline
\end{tabular}

Fonte: Autores (2021).

\section{Evolução da paciente}

Durante o tratamento observou-se uma melhora gradativa do quadro da paciente, já nas primeiras sessões de aplicação da toxina botulínica pode-se perceber uma melhoria na motilidade muscular e redução na assimetria. Ao final das sessões os resultados foram melhorados com o uso de fios de Polidioxanona (PDO) que foram utilizados como alternativa para elevação da região labial, realizando ainda um reposicionamento da musculatura afetada. 
Foi possível observar que a paciente obteve melhora tanto no que tange as funções quando simetria e estética facial, sendo pontos fundamentais de reabilitação em pacientes acometidos pela síndrome de Bell.

\section{Discussão}

A paralisia facial de bell afeta o nervo facial ou VII par craniano sendo o nevo que apresenta paralisia com maior frequência, ela apresenta aspectos fisiopatológicos inerentes a um processo de infecção viral, em relação a clínica este se apresenta já como pior quadro nos primeiros dois dias (Konno, et al., 2014; Bento, et al., 2018; Wenceslau, et al., 2016).

Segundo Silva, et al., (2019), em torno de 50\% da população sofre algum tipo de paralisia facial periférica onde a paralisia de Bell surge com maior incidência.

O diagnóstico é clínico e também de exclusão onde deve-se realizar um exame físico detalhado podendo ainda utilizar exames complementares para auxiliar no diagnóstico, em meio a paralisia de Bell é necessário um olhar integral da equipe multiprofissional visto que a doença afeta muito além do físico, interferindo também na saúde emocional, no ponto que diminui a autoestima (Pedrosa, et al., 2020).

Pedrosa, et al., (2020), demonstra que apesar da indefinição em sua etiologia a paralisia de Bell está amplamente relacionada com infecções, e é comum que a patologia seja confundida inicialmente com o Acidente Vascular Encefálico (AVE), no entanto, percebe-se rapidamente essa diferenciação visto que a paralisia de Bell interfere apenas na parte facial ao contrário do AVE.

O tratamento de tal patologia é bastante diversificado e requer a participação de diferentes profissionais, isso se deve ao fato de que existe diferentes graus da paralisia, bem como diversos fatores etiológicos (Pedrosa, et al., 2020).

Este tratamento busca a recuperação completa da função da musculatura e da mímica facial, prevenindo possíveis sequelas (Andrade, 2019).

Inicialmente o tratamento envolve o uso de medicamentos, métodos físicos e até cirurgia e torna-se bastante eficaz, no entanto, pode-se observar que existe ainda um índice de recuperação incompleta devido ao processo da regeneração neural (Reis, 2021).

A utilização da toxina botulínica consiste em um tratamento auxiliar para pacientes com sequelas da paralisia de Bell, reduzindo significativamente assimetrias (Thien, et al., 2019).

Os conhecimentos acerca da reabilitação facial são fundamentais visto que expressão facial é importante para o auto reconhecimento e comunicação, fatores importantes para se manter uma boa interação social (Reis, 2021).

O uso da toxina botulínica no tratamento é amplamente utilizado devido à capacidade de reduzir assimetrias em repouso e também durante o movimento sendo ainda um opção menos invasiva e de menos riscos, além de não deixar cicatrizes melhorando a estética e em consequência a auto estima da paciente (Andalício, et al., 2021).

Um estudo de revisão integrativa realizado em 2021, avaliou 17 publicações onde foram utilizadas a toxina botulínica no tratamento da paralisia facial periférica dos quais todos apresentaram resultados positivos demonstrando ao fim do tratamento melhora da simetria, hiperatividade muscular e melhora na qualidade de vida dos participantes (Andalício, et al., 2021).

Estudo realizado em 2020, demonstrou que entre os pacientes acometidos pela paralisia facial periférica obtiveram predominância no sexo feminino com idade média de 50,5 anos, onde de acordo com a etiologia da paralisia destacou-se a causa por infecções, no decorrer do estudo observou-se um comprometimento motor acentuado, todavia, este não interferiu na qualidade de vida dos participantes (Medeiros, et al., 2020).

Durante o tratamento faz-se necessário o acompanhamento da evolução do paciente, nesse contexto surge a escala de House-Brackman que consiste em um instrumento para avaliar o grau de evolução (Silva, et al., 2019).

A escala consiste em um instrumento de suma importância visto que permite avaliar o nível de lesão do nervo 
proporcionando um real parâmetro de evolução do paciente durante o tratamento (Silva, et al., 2019). Esta apresenta 4 graus que são apresentados na Tabela 2 a seguir:

Tabela 2: Escala de House-Brackman.

\begin{tabular}{|l|l|}
\hline Grau 1 (Função Normal) & Função Normal \\
\hline Grau 2 (Disfunção) & Ligeira paralisia detectável apenas com uma rigorosa \\
\hline & inspeção \\
\hline \multirow{2}{*}{ Grau 3 (Disfunção Moderada) } & Fecha olho completamente com mínimo esforço \\
\hline & Assimetria só ao sorrir forçadamente \\
\hline & Sem complicações \\
\hline Grau 4 (Disfunção Moderada/Severa) & Paralisia evidente, mas não desfigurante \\
\hline & Fecha olho, mas com grande esforço \\
\hline & Boca com desvio evidente na hora de sorrir \\
\hline & Podem surgir espasmos \\
\hline & Paralisia evidente e desfigurante \\
\hline & Não fecha o olho \\
\hline
\end{tabular}

Fonte: House-Brackman (1985).

Segundo Fonseca, (2015), a escala de House e Brackmann torna-se uma ferramenta importante mediante casos de paralisia, visto que tem fácil interpretação e demonstra os graus de disfunção de forma individual.

Além do uso da TB, no caso relatado foi utilizado fios de PDO durante o tratamento, este teve o objetivo de reposicionar a musculatura afetada devolvendo a simetria facial.

Os fios de PDO tem força por um tempo prolongado e permanecem no tecido pelo tempo necessário, esse tipo de fio tem aprovação da Agência Nacional de Vigilância Sanitária para uso cirúrgico e recentemente tem sido utilizado para lifting facial (Bortolozo, et al., 2021).

O fio de PDO é sintético e absorvível sendo um polímero não alergênico preparado por meio do poliéster, um estudo que avaliou o uso destes fios como técnica para elevação de sobrancelhas demonstrou que esse torna-se uma alternativa economicamente viável com resultados superiores em comparação ao uso de outros fios (Bortolozo, et al., 2021).

A utilização dos fios se tornam cada vez mais uma técnica viável e eficaz em meio a tempos de busca de resultados positivos por meio de procedimentos mais seguros e menos invasivos, sendo estes capazes de suspender os tecidos em um curto período de recuperação (Albuquerque, et al., 2021).

Estudo realizado em 2017 demonstrou o uso dos fios de PDO afim de estimular a produção de colágeno que proporcionou uma melhora na região contra lateral a paralisia (Goldoni et al., 2017).

\section{Considerações Finais}

Percebe-se que a paralisia de Bell consiste em uma doença que ainda não possui etiologia definida sendo em grande parte relacionada a infecções, que afeta tanto a parte física quanto emocional das pessoas acometidas, interferindo significativamente em sua autoestima.

Ademais, a referida patologia possui diagnóstico clínico e de exclusão, e em geral possui um bom prognóstico onde frente a um tratamento adequado possui altos índices de recuperação total, no entanto, há casos em que a paralisia pode provocar sequelas permanentes.

Foi possível perceber que o tratamento alternativo empregado no caso relatado se mostrou bastante eficaz, melhorando 
a estética facial da paciente e sua autoestima o que trouxe um ganho bastante positivo para sua qualidade de vida.

Faz-se necessário novos estudos acerca do uso do presente tratamento afim de comprovar sua eficácia em diferentes situações, uma vez que em a TB já é bastante aceita, no entanto, ainda não há na literatura evidências do uso do fios de PDO no tratamento da Paralisia de Bell.

\section{Referências}

Albuquerque, L. V. de, et al. (2021). Lifting facial não cirúrgico com fios de Polidioxanona: revisão de literatura. Odontol. Clín.-Cient., Recife, 20(1) 39 - 45

Andalécio, M. M. (2021). A utilização da toxina botulínica no tratamento da paralisia facial periférica. Research, Society and Development, $10(9)$, e8510917935.

Andrade, H. M. A. de. (2019). Toxina botulínica e laserterapia associadas ao tratamento da paralisia facial de Bell: Relato de caso clínico. Monografia.

Belém, L. M; et al. (2021). Uso da laserterapia no tratamento de pacientes com paralisia de Bell: revisão crítica da literatura. rev port estomatol med dent cir maxilofac. 62(2):1-6

Bento, R. F., et al. (2018). Tratado de Paralisia Facial: Fundamentos Teóricos -Aplicação Prática / Thieme Revienter Publicações, 294 p.

Brito, A. de S. \& Barbosa, D. B. M. (2020). A utilização da toxina botulínica tipo a para alcançar a estética facial. Rev. Terra \& Cult., 36(70).

Bortolozo, F. (2017). A-PDO - técnica de elevação de sobrancelhas com fios de polidioxanona ancorados - relato de 10 casos. Brazilian Journal of Surgery and Clinical Research 20(1), 76-87.

Fonseca, K. M. (2015). Escalas de grau da paralisia facial: análise de concordância Braz J Otorhinolaryngol. 288-93.

Fujita, R. L. R. \& Hurtado, C. C. N. (20119). Aspectos relevantes do uso da toxina botulínica no tratamento estético e seus diversos mecanismos de ação. Saber Científico, 8(1), $120-133$.

Goldoni, B. I. L., Silva, C. P., \& Rocha, R. V. (2017). Harmonização facial em pacientes com paralisia de bell-relato de caso. Revista Focus In Scientiae.

House J. W. \& Brackmann. (1985). Facial nerve grading system. Otolaryngol Head Neck Surg.93:146-7.

Hugo, A. P. et al. (2020). Evolução clínica de um paciente com síndrome de bell: relato de experiência in A enfermagem centrada na investigação científica 7 [recurso eletrônico] / Organizador Marcus Fernando da Silva Praxedes. Atena, 196-205.

Konno, K. M., et al. (2014). Perfil funcional da paralisia facial em um hospital. Revista Pesquisa Em Fisioterapia, 4(2).

Lima, P. N. de; et al. (2020). Toxina botulínica como alternativa no tratamento da paralisia facial de Bell: revisão de literatura. Brazilian Journal of Development. $6(12), 95667-95681$.

Medeiros, S.F.D, et al. (2020). Bem-estar e comprometimento motor facial em pacientes com paralisia facial periférica: um estudo transversal. Rev Pesqui Fisioter. 10(3):470-477.

Reis, L. do V. (2021). O papel da harmonização orofacial em pacientes sequelados por paralisia parcial periférica - relato de caso clínico. Revista IberoAmericana de Humanidades, Ciências e Educação. 7(8).

Santos, C. F. dos; et al. (2020). Aplicação de toxina botulínica tipo A em paciente com paralisia facial periférica de Bell: relato de caso. RSBO. 17(2):221-5.

Silva, A. B. C.; et al. (2019). Uso da escala de House Breackmann como parâmetro na evolução da paralisia facial periférica: um relato de caso. UniEVANGÉLICA, 1757-1761.

Thien, C. I; et al. (2019). Toxina botulínica no tratamento de sequelas da paralisia facial: área de atuação do dermatologista. Surgical \& Cosmetic Dermatology, 11(3), 238-243

Wenceslau, L. G. C., et al. (2016). Paralisia facial periférica: atividade muscular em diferentes momentos da doença. CoDAS 28(1), 03-09. 